\title{
THE USE OF LESSER-KNOWN THEOREMS AND FORMULAS AS A TOOL FOR OBTAINING ELEGANT SOLUTIONS IN EUCLIDEAN GEOMETRY
}

\author{
MOSHE STUPEL, SHULA WEISSMAN \\ and AVI(BERMAN) SIGLER \\ Gordon and Shaanan Academic College \\ Haifa \\ Israel \\ e-mail: stupel@bezeqint.net \\ Gordon Academic College \\ Haifa \\ Israel \\ e-mail: shulawe@gmail.com \\ Shaanan Academic College \\ Haifa \\ Israel \\ e-mail: avibsigler@gmail.com
}

\begin{abstract}
The methods of teaching and instruction in mathematics are derived from the program of studies and its goals. One of the principal goals of studies of mathematics is to provide the students with methods of reasoning that may assist them in other fields of study and knowledge, and to develop such methods.
\end{abstract}

2010 Mathematics Subject Classification: 97G30, 51N20, 51M25.

Keywords and phrases: lesser-known theorems, elegant solutions, mathematical toolbox. Received February 18, 2019; Revised April 14, 2019

(C) 2019 Scientific Advances Publishers 
The meaning of "learning to think" is: the teacher of mathematics should develop the ability of students to apply information, perform analysis and synthesis at an adequate level, of basic properties, rules and the theorems that they studied at an earlier stage of the teaching process.

By using methods of solution and knowledge from other fields of mathematics one can obtain simpler and faster solutions.

Finding another method of solution by using the same mathematical field, and especially from another field, contributes to development of reasoning and raises it to a higher level.

Implementation of previous knowledge in a new situation that results in a shorter and simpler solution or a more beautiful solution increases enjoyment and satisfaction from studies of the subject.

Integration of fields in problem solution gives the students and wider outlook to mathematics as a comprehensive discipline, while creating connections between its different branches.

The ability to handle different tasks in Euclidean geometry, some of which are hard or complex, depends to a large extent on the "mathematical toolbox" available to student. The wider and richer it is with lesser-known theorems and formulas, the much higher is the student's ability to cope such problems, and it is very likely that they will be able to achieve elegant, short and aesthetic solutions. In order to illustrate the importance of the "mathematical toolbox", we present seven interesting tasks in Euclidean geometry that require theorems and formulas which are not usually known to the students. In six of the tasks we presented more than one solution. Usually the solution obtained by an unknown theorem or formula is surprising, short and the prettiest of all the solutions.

\section{Introduction}

The solution of problems requiring proofs or calculations in geometry necessitates acquaintance with fundamental properties of geometrical shapes, with theorems and various formulas. The more extensive is the "mathematical toolbox" of the student, and the better he is at using these tools, the easier will it be for him to deal with different tasks. The heading "mathematical toolbox" must also include knowledge in other fields of mathematics, such as: algebra, trigonometry, analytic geometry, calculus etc., since the fields of mathematics are intertwining branches of a wide tree. Very often it is possible to solve problems in geometry using 
tools from another branch in mathematics, and the converse is also true. The rich collection of mathematical tools allows one to deal with a certain problem and to find a solution for its using different methods, some of which are very short and surprising, and accentuate the beauty of mathematics. It's enough to mention that hundreds of different proofs were found for the Pythagorean theorem, some of which are similar among them but some are very different.

Each country has its own program of studies, which details the subjects of study and their levels, as well as the set of theorems and formulas that the students must be acquainted with and which are the only ones that they may use for proving and solving problems. On the other hand, in some places mathematical studies are also encouraged by increasing the number of frontal studies and through enrichment courses, courses of mathematical riddles and courses that combine enrichment with investigation accompanied by computer technology. At these places the knowledge of the students is enriched by lesser-known theorems in geometry: the Steiner's theorem for the trapezoid, Ptolemy's theorem for the quadrilateral, Bretschneider's theorem for the quadrilateral, as well as the Stewart, Ceva, Menelaus, Pascal and other theorems regarding formulas for calculating values in certain geometrical shapes, and inequalities between different geometrical magnitudes. Information on theorems, formulas and inequalities which are usually not known to high school students, or to many of their teachers, one can find in enrichment books in geometry and in different papers [1-3]. For example, one can prove the theorem that if two of the angle bisectors in a triangle have equal lengths that the triangle is an isosceles triangle (called the Steiner-Lemus theorem [4, 5]) using formulas and algebraic manipulations.

Those who are taught these enrichment subjects and are familiar with them can attain beautiful and short solutions, tasks that would require the ordinary student to invest much effort and time. Indeed, in 
recent years, in different papers, the importance of the ability to prove or solve a task using different tools or using integration of several tools has been emphasized [6-13].

In order to show how important is knowledge enrichment, we present seven geometrical tasks, for each of which we present two solutions or more, and we have no doubt which of them should be regarded as the preferable and the more beautiful solution.

Task 1: Calculating the area of a triangle using different methods

This task is simple and can be solved using different methods in accordance with the knowledge of the students.

The concept of area is studied at the age of 10-11, following which the students learn to calculate the areas of simple geometrical shapes, including calculating area of a triangle, half the product for a side length and the length of the altitude to that side.

Students at different ages are given the task of calculating the area of a triangle whose side lengths are $13 \mathrm{~cm}, 14 \mathrm{~cm}$, and $15 \mathrm{~cm}$ (see Figure 1).

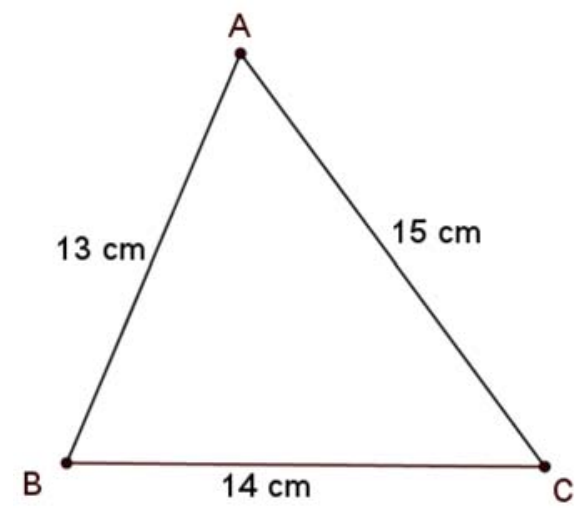

Figure 1. 
(a) Calculating the area of a triangle by students at the age of 10-13

At these ages the students can use a ruler and a right triangle. Using the triangular ruler they draw the altitude $A D$, and measure its length using a ruler, to calculate the area. The accuracy of the result depends on the accuracy of the measurements.

(b) Calculating the area of a triangle by students at the age of 14-15

At these ages the students are familiar with the Pythagorean theorem and can solve quadratic equations. They draw the altitude $A D$ and denote:

$B D=x$, and $D C=14-x$ (see Figure 2). Two quadratic equations are obtained:

$$
\left.\begin{array}{l}
(A D)^{2}=13^{2}-x^{2} \\
(A D)^{2}=15^{2}-(14-x)^{2}
\end{array}\right\} \Rightarrow A D=12 \mathrm{~cm} .
$$

Hence, $S=84 \mathrm{~cm}^{2}$.

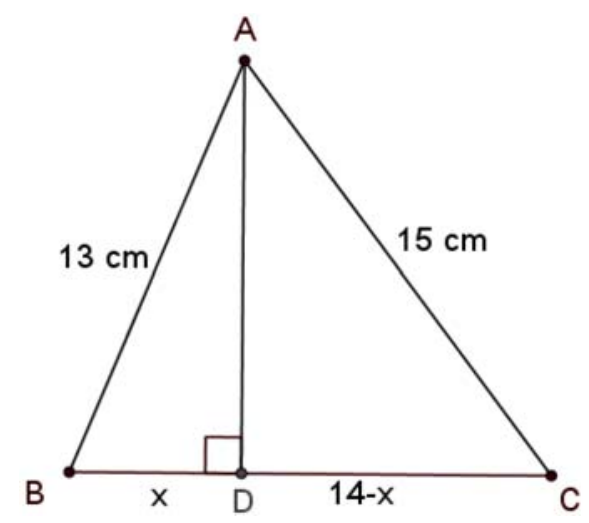

Figure 2. 
(c) Calculating the area of a triangle by students at the age of 16-18

These students know trigonometry, and using the law of cosines they find the magnitude of one of the angles, and using the formula $S=0.5 \cdot a \cdot b \cdot \sin \gamma$ they find the area of the triangle.

\section{(d) Calculating the area of a triangle by Heron's formula}

Heron's formula for calculating the area of triangle is $S=\sqrt{p(p-a)(p-b)(p-c)}$, where $a, b$, and $c$ are the sides of the triangle, and $p$ is half of its perimeter $\left(p=\frac{a+b+c}{2}\right)$.

This formula allows one to calculate the area of the triangle by students of any age who can substitute in the formula to calculate the numeric value of the obtained expression. There is no need to measure the altitude, to use the Pythagorean theorem or the law of the cosines. The presentation of the fourth method accentuates the fact that acquaintance with an algebraic formula allows one to obtain the value of the area in the shortest possible way.

Note: When the triangle is given in the system of coordinates and one is to calculate the area, clearly calculating the area using a determinant is better than a geometrical method, or a method from analytical geometry or a combination of analytical geometry and trigonometry.

Task 2: Calculating the length of the segment that connects the midpoints of a trapezoid's bases

Given is a trapezoid $A B C D$, the sum of whose base angles is $\alpha+\beta=90^{\circ}$, and the lengths of whose bases are: $A B=a$ and $D C=b$, $a>b$ (see Figure 3). 


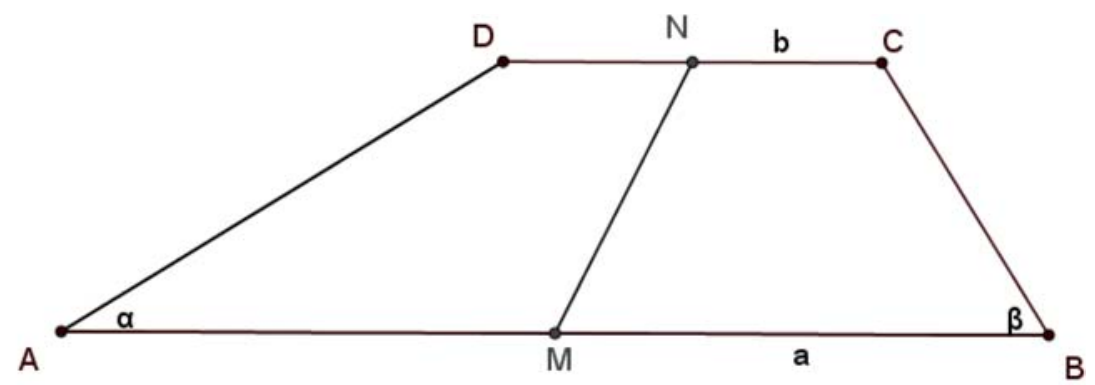

Figure 3.

Express the length of the segment $M N$ where the points $M$ and $N$ are the middles of the lower base and the upper base, respectively.

Method A (Geometry with an auxiliary construction):

Construct $N E\|C B, N F\| D A$, two parallelograms are formed, $A D N F$ and $N C B E$, as shown in Figure 4. Calculation gives $F E=a-b$. The triangle $F N E$ is a right-angled triangle, i.e., $\angle F N E=90^{\circ}, N M$ is a median to its hypotenuse and therefore $M N=\frac{a-b}{2}$.

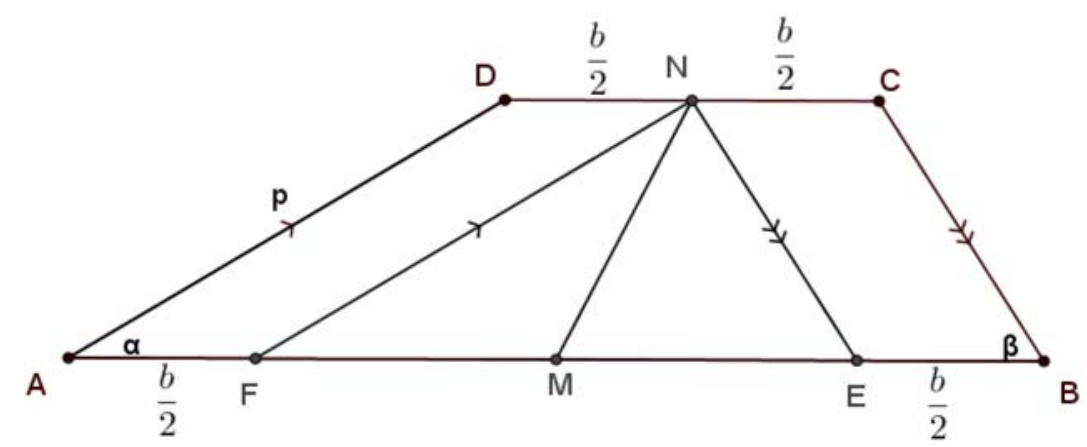

Figure 4 .

Method B (Geometry with Steiner's theorem):

The continuations of the sides intersect at the point $G$ (see Figure 5). According to Steiner's theorem [14]: the midpoints of the trapezoid's bases, the point of intersection of the diagonals of the trapezoid and the 
point of intersection of the continuations of its sides lie on a single straight line. Therefore, since $\angle G=90^{\circ}, G N$ is a median to the hypotenuse in the triangle $\triangle G D C$ and $G M$ is a median to the hypotenuse in the triangle $\triangle G A B$, we obtain

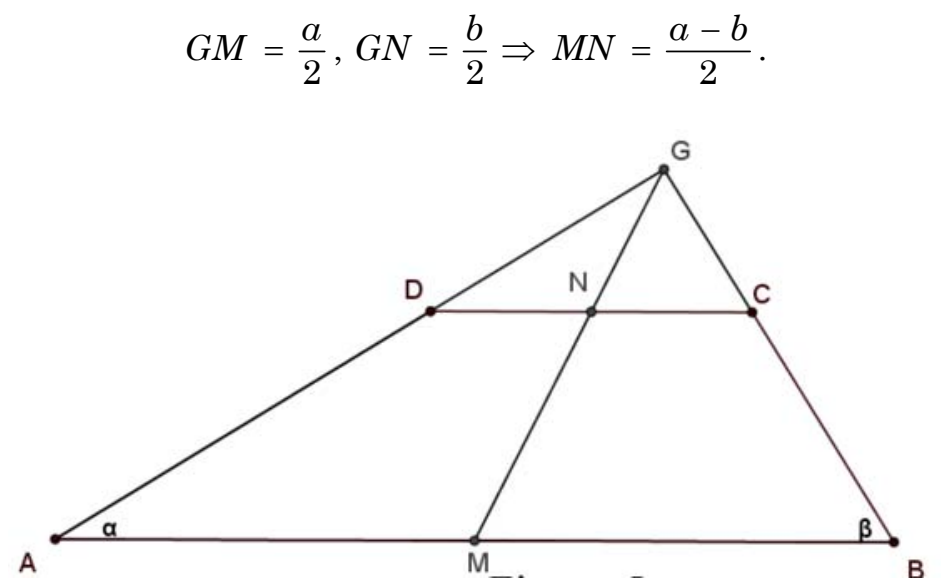

Figure 5.

\section{The converse theorem:}

Given is a trapezoid $A B C D$, whose base lengths are: $A B=a$ and $D C=b$. It is also given that the length of the segment that connects the midpoints of its bases, $M$ and $N$ is $M N=\frac{a-b}{2}$ as described in Figure 3. Prove that the sum of the angles by the lower base is $\alpha+\beta=90^{\circ}$.

Method A (Geometry):

Based on the construction described in Method A (Figure 4), after parallels are drawn to the sides, we obtain $F M=M E=\frac{a-b}{2}$ and since it is given that $M N=\frac{a-b}{2}, M N$ is a median to the side $F E$ in the triangle $N F E$, and it equals half its length; therefore the triangle is right- 
angled, $\angle F N E=90^{\circ}$. Therefore, $\angle N F E+\angle N E F=90^{\circ}$. Hence, since the auxiliary lines $N F$ and $N F$ are parallel to the sides of the trapezoid, it follows that the sum of its base angles is $90^{\circ}$.

Method B (Using Steiner's theorem):

From Steiner's theorem and based on the notation in Figure 5, there holds: $G M-G N=\frac{a-b}{2}$ and $\frac{G N}{G M}=\frac{b}{a}$ (similarity of triangles).

From these two relations we obtain $G N=\frac{b}{2}$.

$G N$ is a median to the side $D C$ in the triangle $G D C$, and it equals half the length of the side $D C$. Therefore $\angle C G D=90^{\circ}$ and the sum of the base angles of the trapezoid is $90^{\circ}$.

\section{Task 3: Conservation property of chord lengths}

Given is an equilateral triangle $A B C$ inscribed in a circle. We draw some chord $B D$, prove that $B D=A D+D C$ (see Figure 6).

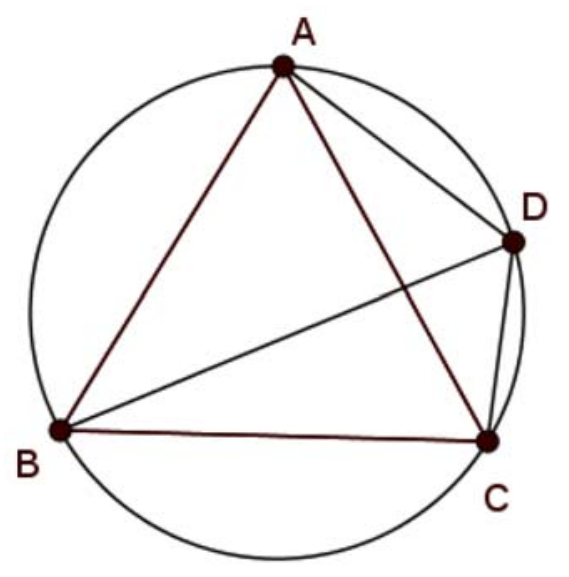

Figure 6. 
To illustrate the property of conservation of the sum of the lengths of the two chords as the length of the third chords we prepared a Geo Gebra applet in which one can drag the point $D$ on the circular arc, thus changing the lengths of the three chords. At each stage the lengths of the three chords and the sum of the chord lengths $A D+D C$ appear on the applet screen. The applet can be reached by the link: Link: https://www.geogebra.org/m/dwybrhe9

Method A (Geometry using auxiliary construction):

On the chord $B D$ we mark a point $E$, so that $A D=D E$ (see Figure 7). We denote: $\angle A B D=\alpha$ and use the theorem: inscribed angles that rest on the same arc are equal. The obtained triangle $A E D$ is an equilateral triangle (an isosceles triangle with a vertex angle of $60^{\circ}$ ). The triangles $A B E$ and $A D C$ are equal at the angle $\alpha$ (inscribed angle), and have an angle of $120^{\circ}(\angle A F B=\angle A D C)$, and therefore they are also equal at the third angle. In addition, they have an equal side $a$, and therefore, based on angle-side-angle, they are congruent. From the congruence, it follows that $E B=D C$. Hence, $B D=D E+E B=A D+D C$.

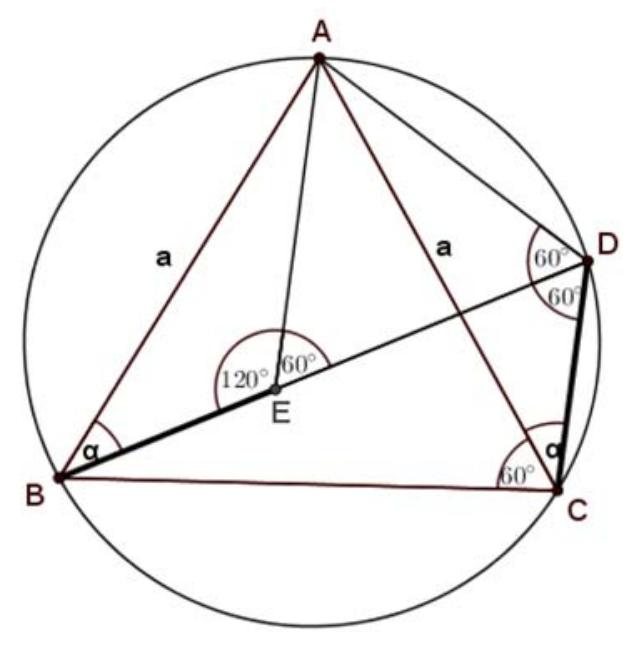

Figure 7. 
Method B (The law of sines):

By using the law of sines in the triangle $B D C$, we obtain:

$$
\begin{aligned}
& \frac{B D}{\sin (60+\alpha)}=\frac{a}{\sin 60} \Rightarrow B D=\frac{2 a}{\sqrt{3}} \sin (60+\alpha), \\
& \frac{D C}{\sin (60-\alpha)}=\frac{a}{\sin 60} \Rightarrow D C=\frac{2 a}{\sqrt{3}} \sin (60+\alpha) .
\end{aligned}
$$

Using the law of sines in the triangle $A B D$ gives:

$$
\frac{A D}{\sin \alpha}=\frac{a}{\sin 60} \Rightarrow A D=\frac{2 a}{\sqrt{3}} \sin \alpha
$$

Hence

$$
\begin{array}{r}
A D+D C=\frac{2 a}{\sqrt{3}}[\sin (60-\alpha)+\sin \alpha]=\frac{2 a}{\sqrt{3}} \cdot 2 \sin 30 \cos (30-\alpha)=\frac{2 a}{\sqrt{3}} \sin \\
(60+\alpha)=B D .
\end{array}
$$

Method C (Trigonometry - The law of cosines):

Using the law of cosines in the triangle $A D B$ gives:

$$
a^{2}=(A D)^{2}+(B D)^{2}-2 A D \cdot B D \cos 60^{\circ}=(A D)^{2}+(B D)^{2}-A D \cdot B D .
$$

Using the law of cosines in the triangle $B D C$ gives:

$$
a^{2}=(D C)^{2}+(B D)^{2}-2 D C \cdot B D \cos 60^{\circ}=(D C)^{2}+(B D)^{2}-D C \cdot B D .
$$

Subtracting the two relations:

$$
0=(A D)^{2}-(D C)^{2}-B D(A D-D C)=(A D-D C)(A D+D C-B D) .
$$

If $A D-D C=0$, then $A D=D C$ and therefore $\angle A B D=\angle D B C=30^{\circ}$.

The triangles $B D C$ and $B A D$ have angles of $30^{\circ}, 60^{\circ}$ and $90^{\circ}$, and therefore it holds that $B D=2 D C=2 A D$ or $B D=A D+D C$. QED. If $A D+D C-B D=0$, then $B D=A D+D C \cdot \mathrm{Q} E D$. 
Before we present the fourth method, we add to the toolbox Ptolemy's theorem $[1,2]$.

Ptolemy's theorem: In every quadrilateral there holds $a c+b d \geq$ $m n$, where $a, c$ are a pair of opposite sides, $b, d$ are the other pair of opposite sides, and $m, n$ are the diagonals of the quadrilateral. Equality holds when the quadrilateral $A B C D$ is a cyclic quadrilateral (a quadrilateral that can be inscribed in a circle).

Method D (Using Ptolemy's theorem):

The quadrilateral $A B C D$ is inscribed in a circle, therefore there holds

$$
\begin{gathered}
A B \cdot D C+A D \cdot B C=B D \cdot A C \\
\Downarrow \\
a \cdot D C+A D \cdot a=B D \cdot a \\
\Downarrow \\
D C+A D=B D
\end{gathered}
$$

We presented 4 solutions using different methods for this task. The reader is invited to choose the simplest and most beautiful solution. It is likely that he will choose the solution $D$, which does not require an auxiliary construction or trigonometric knowledge.

\section{Task 4: Calculating the upper base length of a trapezoid}

Given is an isosceles trapezoid whose base length is $a=12 \mathrm{~cm}$, whose side length is $c=2 \sqrt{7} \mathrm{~cm}$, and whose diagonal length is $k=10 \mathrm{~cm}$. Calculate the length of its other base $b$ (see Figure 8). 


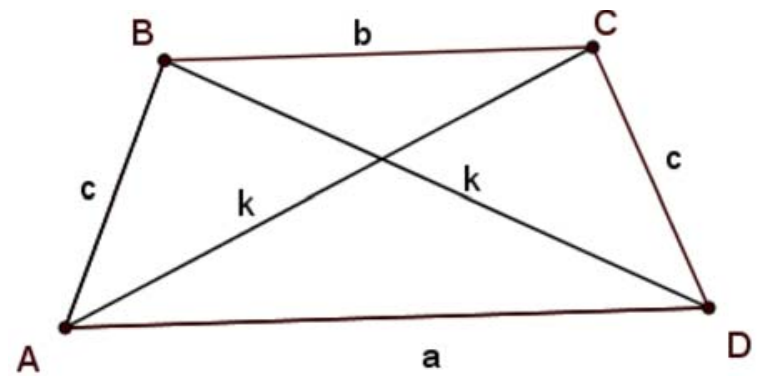

Figure 8.

Method A (Using trigonometry):

We denote: $\angle B A D=\alpha$. By using the law of cosines in the triangle $\triangle B A D$, we obtain $\cos \alpha=\frac{3}{2 \sqrt{7}}$. We construct $B E$ in such a manner that $B E \| C D$ (see Figure 9).

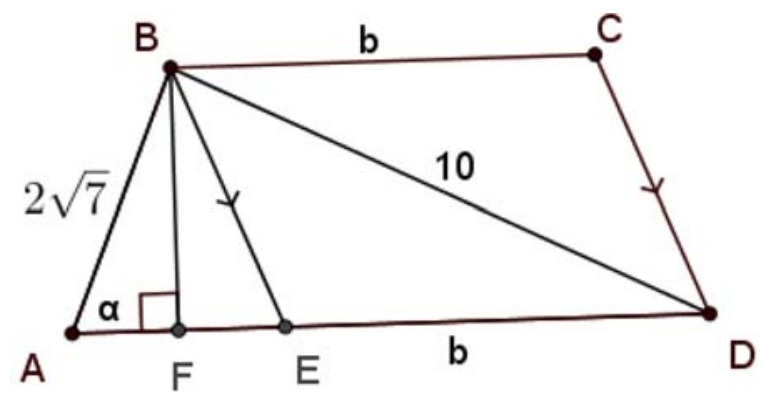

Figure 9.

An isosceles triangle $\triangle B A E$ is formed $(A B=B E)$. In this triangle, we construct the altitude $B F$ and obtain $A F=\frac{a-b}{2}$. By representing $\cos \alpha$ from the triangle $\triangle B A F$, we obtain

$$
\cos \alpha=\frac{A F}{A B}=\frac{\frac{a-b}{2}}{2 \sqrt{7}} \Rightarrow a-b=6 \Rightarrow b=6 .
$$


Method B (Using the Pythagorean theorem):

From the considerations mentioned in Method A we obtain $A F=\frac{a-b}{2}, F D=\frac{a+b}{2}$. From the Pythagorean theorem in the triangle $\triangle B A F$, we obtain $(B F)^{2}=(2 \sqrt{7})^{2}-\left(\frac{a-b}{2}\right)^{2}$. From the Pythagorean theorem in the triangle $\triangle B D F$, we obtain $(B F)^{2}=10^{2}-\left(\frac{a+b}{2}\right)^{2}$. By comparing the two expressions, we obtain $a b=72 \Rightarrow b=6 \mathrm{~cm}$.

Method C (Using Ptolemy's theorem):

The given trapezoid can be inscribed in a circle because it is an isosceles trapezoid (see Figure 10). From Ptolemy's theorem, we have $c^{2}+a b=k^{2}$.

Hence,

$$
b=\frac{k^{2}-c^{2}}{a}=\frac{100-28}{12}=6 \mathrm{~cm} .
$$

It is easy to decide which is the most elegant and simple solution of the three solutions presented.

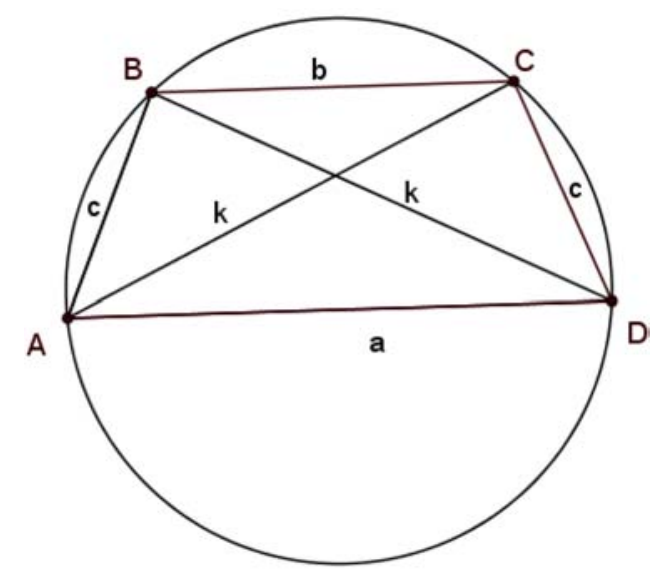

Figure 10. 
Task 5: Mathematical relation between the sides and the diagonals of a parallelogram

Given is a parallelogram $A B C D$ with an acute angle of $45^{\circ}$. Prove that the product of the squares of its diagonals equals the sum of the fourth powers of the sides of the parallelogram. In other words, prove that $(A C)^{2} \cdot(B D)^{2}=a^{4}+b^{4}$ (see Figure 11).

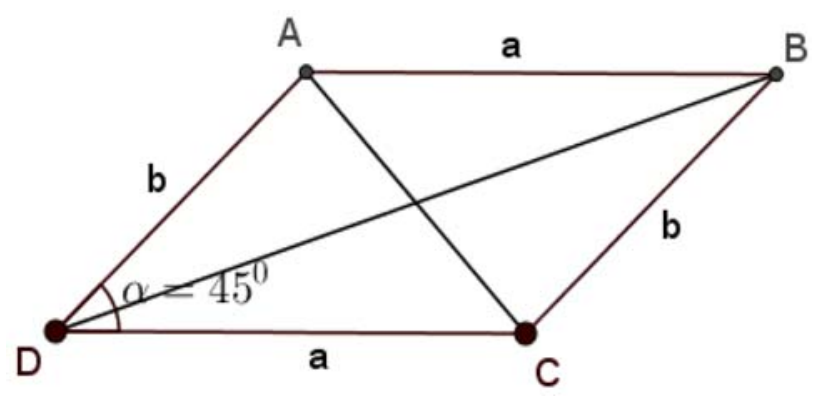

Figure 11.

Method A (Using the law of cosines):

$$
\begin{aligned}
&(A C)^{2}=a^{2}+b^{2}-2 a b \cos 45^{\circ}=a^{2}+b^{2}-\sqrt{2} a b, \\
&(B D)^{2}=a^{2}+b^{2}-2 a b \cos 135^{\circ}=a^{2}+b^{2}+\sqrt{2} a b, \\
&(A C)^{2} \cdot(B D)^{2}=\left(a^{2}+b^{2}-\sqrt{2} a b\right)\left(a^{2}+b^{2}+\sqrt{2} a b\right)=\left(a^{2}+b^{2}\right)^{2}-2 a^{2} b^{2} \\
&=a^{4}+b^{4} .
\end{aligned}
$$

Method B (Using the Bretschneider's theorem):

To enrich the mathematical toolbox we present Bretschneider's theorem [15]: For every convex quadrilateral whose side lengths are $a, b$, $c, d$, whose diagonals are $g$ and $f$, and two of whose opposite angles are $\angle A, \angle C$ (see Figure 12) there holds,

$$
g^{2} \cdot f^{2}=a^{2} \cdot c^{2}+b^{2} \cdot d^{2}-2 a b c d \cos (\angle A+\angle C) .
$$


The given problem is a particular case of the Bretschneider's theorem. Here the opposite sides are equal and the sum of the opposite angles is $90^{\circ}$. Therefore, we obtain $g^{2} \cdot f^{2}=a^{4}+b^{4}$.

Note: Ptolemy's theorem is a particular case of the Bretschneider's theorem for a quadrilateral that can be inscribed in a circle (which satisfies: $\angle A+\angle C=180^{\circ}$ ).

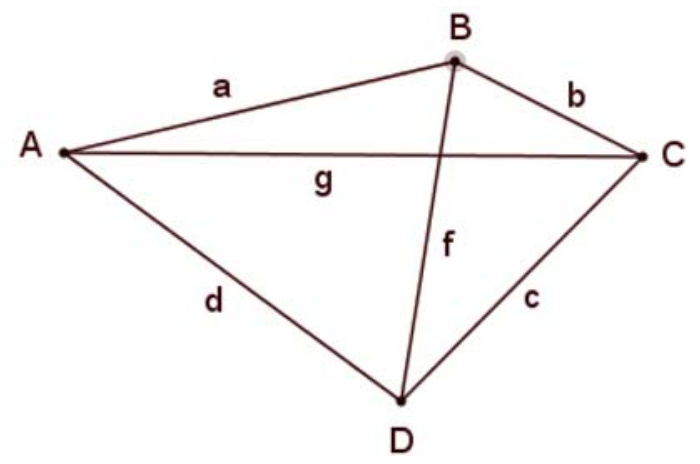

Figure 12.

\section{Task 6: Calculating the side length of an isosceles triangle}

Given is an isosceles triangle $\triangle A B C(A B=A C) . D$ is a point on the base $B C$ so that $B D=4 \mathrm{~cm}, D C=10 \mathrm{~cm}$. It is also given that $A D=9 \mathrm{~cm}$. Find the length of the side of the triangle (see Figure 13).

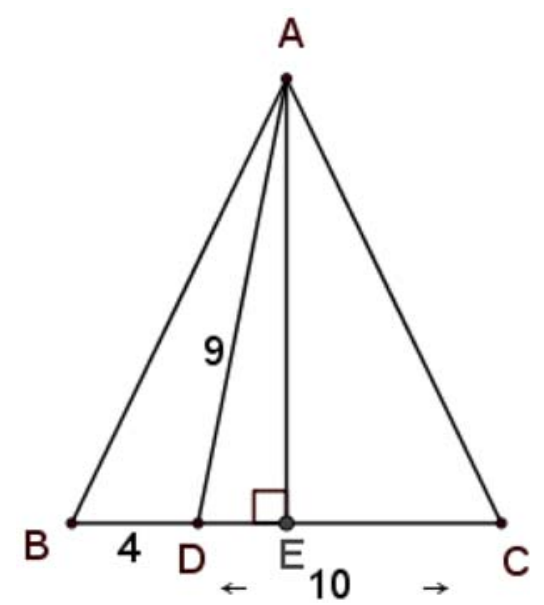

Figure 13. 
Method A (Using the Pythagorean theorem):

Construct the altitude $A E$ to the base and calculate $D E=3 \mathrm{~cm}$. From the Pythagorean theorem in the triangle $\triangle A D E$, we obtain $A E=\sqrt{72} \mathrm{~cm}$. From the Pythagorean theorem in the triangle $\triangle A B E$, we obtain $A B=A C=11 \mathrm{~cm}$.

Method B (Using the law of cosines):

We denote $\angle A D B=\delta$ (see Figure 14). From the law of cosines in the triangles $\triangle A D C, \triangle A B D$, we obtain

$$
\begin{aligned}
& (A B)^{2}=4^{2}+9^{2}-2 \cdot 4 \cdot 9 \cos \delta \\
& (A C)^{2}=10^{2}+9^{2}-2 \cdot 10 \cdot 9 \cos (180-\delta)=10^{2}+9^{2}+2 \cdot 10 \cdot 9 \cos \delta .
\end{aligned}
$$

By extracting $\cos \delta$ from the first equation and substituting it into the second one we obtain $A B=11 \mathrm{~cm}$.

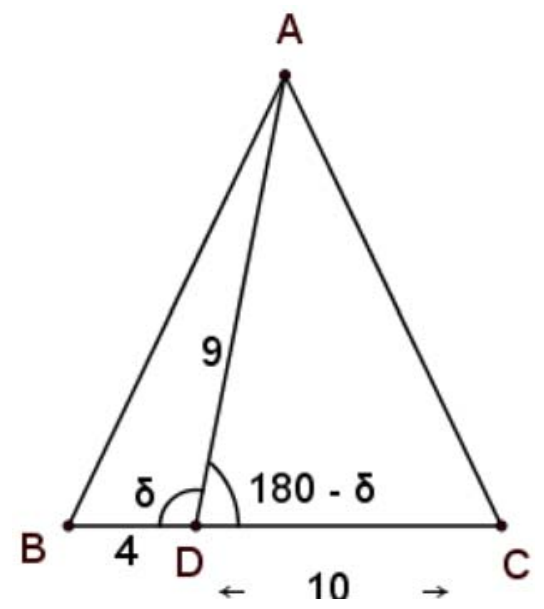

Figure 14. 
Method C (Using the Hoehn's theorem (discovered in the year 2000)):

From the Hoehn's theorem [16], for an isosceles triangle there holds:

$$
(A B)^{2}=(A D)^{2}+B D \cdot D C
$$

where $A D$ is a segment that issues from the vertex angle to the base (see Figure 15). By substituting the data in this theorem, we obtain

$$
(A B)^{2}=9^{2}+4 \cdot 10=121 \Rightarrow A B=11 \mathrm{~cm} .
$$

This theorem allows us to find one of the values immediately, if the other three values are given.

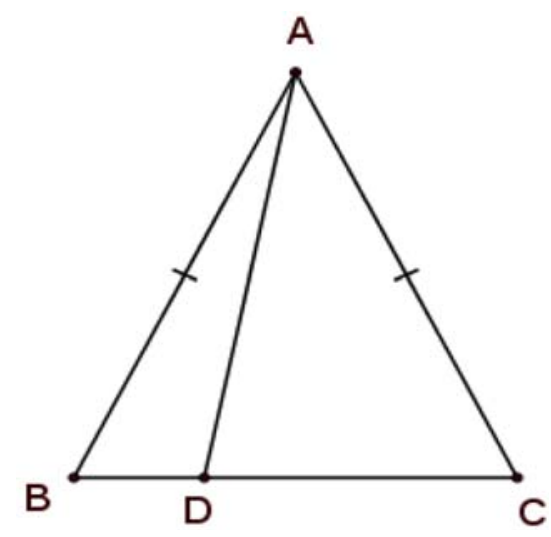

Figure 15.

\section{Task 7: A quadrilateral in which questions arise progressively}

In this task, in order to solve the questions, the information base of the solving person must be supplemented at each stage. Given is a convex quadrilateral in which $a$ and $c$ are a pair of opposite sides. The lengths of the other pair of opposite sides are $b$ and $d$, and the lengths of its diagonals are $m$ and $n$. Let $O$ be the point of intersection of the diagonals (see Figure 16). 


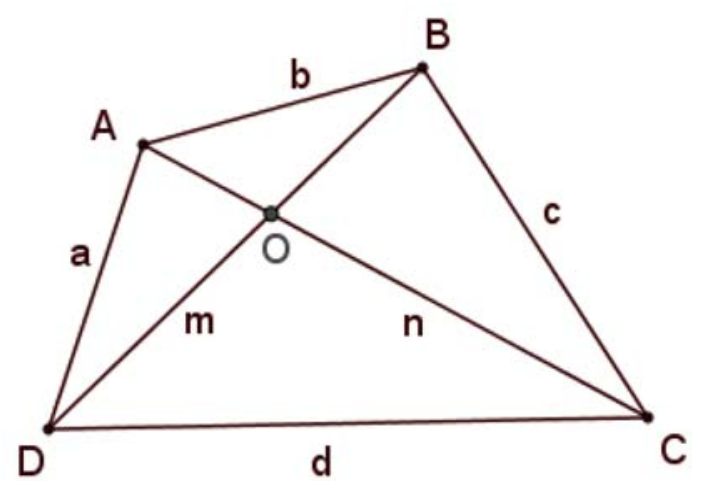

Figure 16.

\section{Question A}

Prove that the lengths of the segments $a+c, b+d$ and $m+n$ satisfy the triangle inequality (the sum of the lengths of each pair of sides is larger than the length of the third side).

Stage 1: Prove that $m+n>a+c$.

$$
\left.\begin{array}{l}
m=D O+O B \\
n=A O+O C
\end{array}\right\} \Rightarrow m+n=(D O+A O)+(O B+O C) .
$$

From the triangle inequality in the triangle $\triangle A O D$, we obtain that $D O+A O>a$.

From the triangle inequality in the triangle $\triangle B O C$, we obtain that $O B+O C>a$.

Hence, $m+n>a+c$.

Stage 2: We prove that $m+n>b+d$ in the same manner that we proved Stage 1. 
Stage 3: We prove that $(a+b)+(b+d)>(m+n)$.

From the triangle inequality in the triangle $\triangle A B D$, we obtain that $a+b>m$.

From the triangle inequality in the triangle $\triangle B C D$, we obtain that $c+d>m$.

Hence, $a+b+c+d>2 m$.

From the triangle inequality in the triangle $\triangle A B C$, we obtain that $b+c>n$.

From the triangle inequality in the triangle $\triangle A C D$, we obtain that $a+d>n$.

Hence, $a+b+c+d>2 n$. Hence it follows that

$$
\begin{gathered}
2(a+b)+2(b+d)>2(m+n) \\
\Downarrow \\
(a+b)+(b+d)>(m+n) .
\end{gathered}
$$

Before presenting Questions B and C, we add two theorems to the mathematical toolbox: The generalized Euler's theorem for the quadrilateral and the law of cosines for the quadrilateral.

\section{The generalized Euler's theorem for the quadrilateral}

Let $M$ and $N$ be the midpoints of the diagonals $B D$ and $A C$, respectively (as shown in Figure 17(a)), and the midpoints of the opposite sides (as shown in Figure 17(b) and (c)), which are required for writing down the relations between the lengths of the sides of the quadrilateral, the lengths of its diagonals and the distance between the midpoints $M$ and $N$, in accordance with the generalized Euler's theorem for the quadrilateral [17]. 


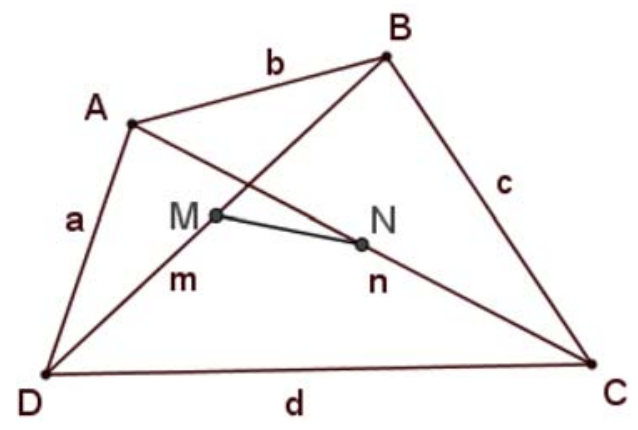

(a)

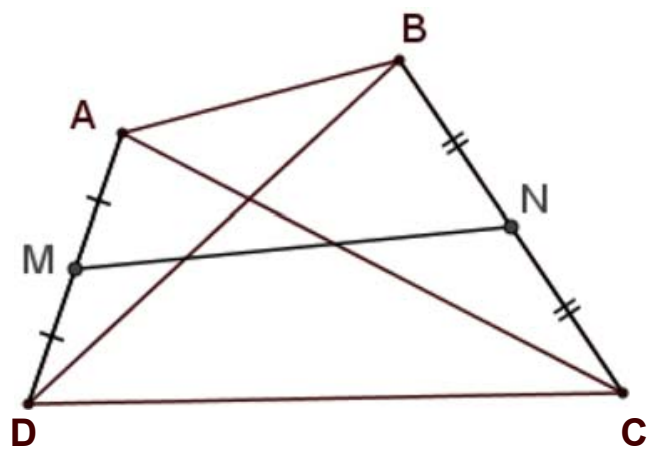

(b)

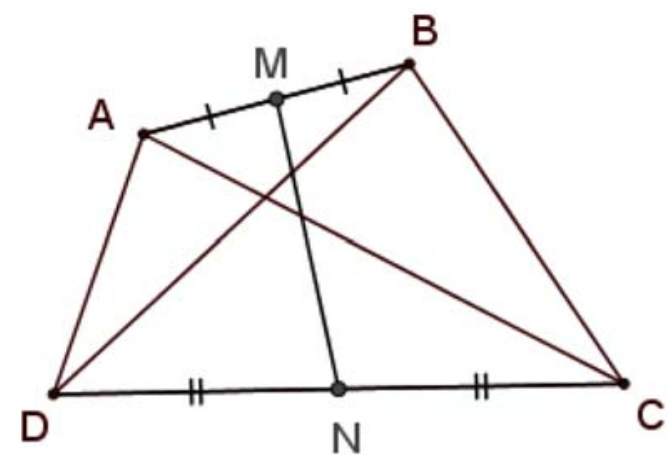

(c)

Figure 17. 
According to the theorem, the relations (1)-(3) hold:

$$
\begin{aligned}
& \left(a^{2}+c^{2}\right)+\left(b^{2}+d^{2}\right)=m^{2}+n^{2}+4(M N)^{2} \\
& \left(b^{2}+d^{2}\right)+\left(m^{2}+n^{2}\right)=a^{2}+c^{2}+4(M N)^{2} \\
& \left(a^{2}+c^{2}\right)+\left(m^{2}+n^{2}\right)=b^{2}+d^{2}+4(M N)^{2}
\end{aligned}
$$

\section{The law of cosines for the quadrilateral}

The law states that $\left(a^{2}+c^{2}\right)-\left(b^{2}+d^{2}\right)=2 m n \cos \alpha$, where the angle $\alpha$ is the angle between the diagonals (see Figure 18).

Note: A student who knows the law of cosines for the triangle will be able to prove the law of cosine for the quadrilateral quite easily.

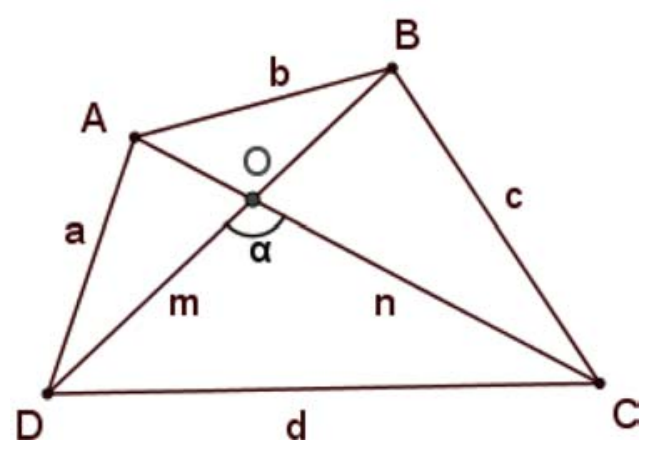

Figure 18.

\section{Question B}

Given the same data, one must prove that the segments whose lengths are $\sqrt{m^{2}+n^{2}}, \sqrt{b^{2}+d^{2}}, \sqrt{a^{2}+c^{2}}$ form an acute triangle.

Clearly the lengths of these segments satisfy the triangle inequality, and it remains to prove that its angles are smaller than $90^{\circ}$. 
The following inequalities arise from the three relations of the generalized Euler's theorem for the quadrilateral:

$$
\begin{aligned}
& \left(\sqrt{a^{2}+c^{2}}\right)^{2}+\left(\sqrt{b^{2}+d^{2}}\right)^{2}-\left(\sqrt{m^{2}+n^{2}}\right)^{2}>0 \\
& \left(\sqrt{b^{2}+d^{2}}\right)^{2}+\left(\sqrt{m^{2}+n^{2}}\right)^{2}-\left(\sqrt{a^{2}+c^{2}}\right)^{2}>0 \\
& \left(\sqrt{a^{2}+c^{2}}\right)^{2}+\left(\sqrt{m^{2}+n^{2}}\right)^{2}-\left(\sqrt{b^{2}+d^{2}}\right)^{2}>0 .
\end{aligned}
$$

Each of the three inequalities represents the cosine of one of the angles of the triangle, and since each of the expressions is positive, all the angles of the triangle are acute.

Note 1: The segments form an acute triangle, aside for the case in which the quadrilateral $A B C D$ is a parallelogram (square, rectangle, rhombus), in which case a right-angled triangle is formed because the length of the segment between the midpoints of the diagonals is $M N=0$.

Note 2: The question can be proven by means of the law of cosines for the quadrilateral.

\section{Question C}

Prove that $a+c, b+d, m+n$ form an acute-angled triangle.

Proof. Based on Euler's formula: $\left(a^{2}+c^{2}\right)+\left(b^{2}+d^{2}\right)>m^{2}+n^{2}$. From Ptolemy's theorem, $2 a c+2 b d \geq 2 m n$.

By combining the two inequalities we obtain $(a+c)^{2}+(b+d)^{2}>$ $(m+n)^{2}$. However, in Question A, we proved that $m+n>a+c$ and $m+n>b+d$. 
In other words, $m+n$ is the longest side in the triangle, and opposite it lies the largest angle $\angle P Q T$ (as shown in Figure 19), and therefore: $(a+c)^{2}+(b+d)^{2}-(m+n)^{2}>0$.

Hence, based on the law of cosines for the triangle $\triangle P Q T, \cos \angle P Q$ $T>0$, in other words the largest angle in the triangle is an acute angle.

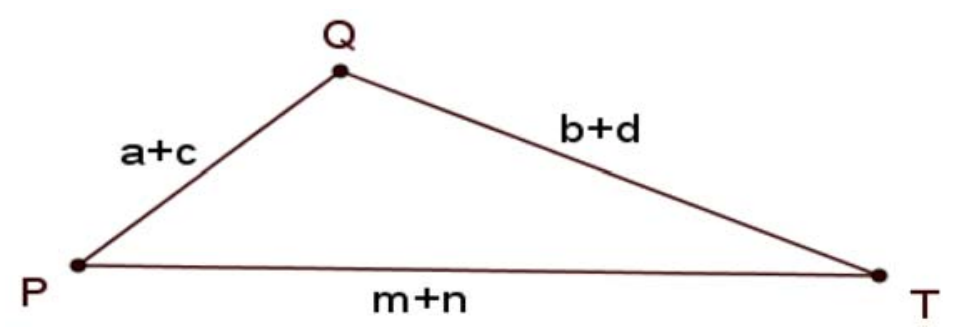

Figure 19.

\section{Summary}

We presented seven tasks that illustrate the importance and the large contribution of acquaintance with lesser-known theorems and formulas, the use of which for proofs and for solving problems in Euclidean geometry allows one to deal with them more easily and to obtain elegant, beautiful and short solutions.

\section{References}

[1] D. E. Smith, A Source Book in Mathematics, Dover Ed., New York, 1959.

[2] H. S. M. Coxeter and Samuel L. Greitzer, Geometry Revisited, Mathematical Association of America, Washington, DC, 1967.

[3] B. Grünbaum and G. C. Shephard, Ceva, Menelaus, and the Area Principle, Mathematics Magazine 68(4) (1995), 254-268.

DOI: https://doi.org/10.1080/0025570X.1995.11996330 
[4] C. F. Parry, A variation on the Steiner-Lehmus theorem, The Mathematical Gazette 62(420) (1978), 89-94.

DOI: https://doi.org/10.2307/3617662

[5] D. Beran, SSA and the Steiner-Lehmus theorem, The Mathematics Teacher 85(5) (1992), 381-383.

[6] M. Stupel and D. Ben-Chaim, One problem, multiple solutions: How multiple proofs can connect several areas of mathematics, Far East Journal of Mathematical Education 11(2) (2013), 129-161.

[7] A. Levav-Waynberg and R. Leikin, Multiple Solutions for a Problem: A Tool for Evaluation of Mathematical Thinking in Geometry, In Proceedings of CERME 6,

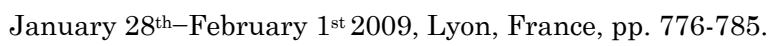

[8] R. Liekin, Multiple proof tasks: Teacher practice and teacher education, ICME Study 19(2) (2009), 31-36.

[9] M. Stupel and D. Ben-Chaim, Using multiple solutions to mathematical problems to develop pedagogical and mathematical thinking: A case study in a teacher education program, The Investigations in Mathematics Learning 9(2) (2017), 86-108.

DOI: https://doi.org/10.1080/19477503.2017.1283179

[10] S. Weissman, M. Stupel and A. Sigeler, Increasing the usage of the area for enriching the toolbox of problem solution and proof in mathematics, Far East Journal of Mathematical Education 18(1) (2018), 1-20.

DOI: http://dx.doi.org/10.17654/ME018010001

[11] D. Fraivert and M. Stupel, Adapting the Formulas Relating the Side of a Triangle with Special Segments in a Triangle and Making them More Accessible, as a Tool for Proofs and Tasks in Mathematics, In the at Right Angles - Mathematics (July Issue) (2018), 71-84.

[12] M. Stupel and V. Oxman, Integrating various fields of mathematics in the process of developing multiple solutions to the same problems in geometry, Australian Senior Mathematics Journal 32(1) (2018), 19-33.

[13] R. Leikin, Openness and constraints associated with creativity-directed activities in mathematics for all students, Broadening the Scope of Research on Mathematical Problem Solving, Springer, Cham (2018), 387-397.

DOI: https://doi.org/10.1007/978-3-319-99861-9_17

[14] M. Stupel and D. Ben-Chaim, A fascinating application of Steiner's theorem for trapezium: Geometric constructions using straightedge alone, Australian Senior Mathematics Journal 27(2) (2013), 6-24. 
[15] R. Mogilevsky and M. Stupel, Forgotten theorems in geometry of the plane and illustration of the use thereof for solving problems (in Hebrew), Shaanan: A Multidisciplinary Journal for Study and Research 10 (2005), 231-252.

[16] L. Hoehn, A neglected Pythagorean-like formula, The Mathematical Gazette 84(499) (2000), 71-73.

$$
\text { DOI: https://doi.org/10.2307/3621478 }
$$

[17] Geoffrey A. Kandall, Euler's theorem for generalized quadrilaterals, College Mathematics Journal 33(5) (2002), 403-404. 